\title{
Effects of enhanced environment and induced depression on cuprizone mouse model of demyelination
}

\author{
AYMAN MOHAMED ${ }^{1}$, GHADA AL-KAFAJI ${ }^{2}$, AMAL ALMAHROOS ${ }^{1}$, \\ ZAHRA ALMOSAWI $^{1}$, HAWRA ALALWAN ${ }^{1}$, REEM ABDULLA $^{1}$, \\ FAJER ALAMMADI ${ }^{1}$, AHMED ALMUBARAK ${ }^{1}$, AHMED AL-MAHREZI $^{1}$ and AMER KAMAL ${ }^{1}$ \\ Departments of ${ }^{1}$ Physiology and ${ }^{2}$ Molecular Medicine and Al-Jawhara Centre for Molecular Medicine, \\ College of Medicine and Medical Sciences, Arabian Gulf University, Manama Block 329, Kingdom of Bahrain
}

Received August 28, 2018; Accepted May 30, 2019

DOI: $10.3892 /$ etm.2019.7654

\begin{abstract}
Impairment in cognition and motor activity are commonly encountered in patients affected by multiple sclerosis (MS), and depression is believed to be a contributing factor. The aim of the present study was to investigate the impact of induced depression on a cuprizone mouse model of demyelination and the effectiveness of enhanced environment (EE) as a method of intervention. C57BL/6 male mice were divided into five groups: Cuprizone only (Cup-O), cuprizone undergoing depression (Cup-Dep), cuprizone housed in EE (Cup-EE), cuprizone housed in EE and undergoing depression (Cup-ED) and the control ( $\mathrm{n}=9-10$ per group). Depression was induced by repeated open-space forced swim. Neurobehavioral tests were conducted following a 6 -week period of $0.2 \%$ cuprizone-enriched diet. The Cup-EE group performed significantly better in terms of cognition and motor functions, when compared with the Cup-O group, as evidenced by the Morris water maze (MWM; $\mathrm{P}<0.001)$ and rotarod performance test $(\mathrm{P}<0.05)$ results. Conversely, the Cup-Dep group exhibited a significant decline in performance in the MWM $(\mathrm{P}<0.001)$ and rotarod performance test $(\mathrm{P}<0.05)$, when compared with the Cup-O group. The Cup-ED group had comparable results to those of the Cup-O group, indicating a reversal of the induced depression effects. Open field test results failed to show an anxiety-like behavior in the cuprizone mouse model. It was therefore concluded that EE can improve MS-associated cognitive and motor deficits. Insights gained from these results facilitate the exploration of non-medical modes of intervention as an emerging adjuvant therapy in MS.
\end{abstract}

Correspondence to: Dr Ghada Al-Kafaji, Department of Molecular Medicine and Al-Jawhara Centre for Molecular Medicine, College of Medicine and Medical Sciences, Arabian Gulf University, 293 Salmaniya Avenue, Manama Block 329, Kingdom of Bahrain E-mail: ghadaa@agu.edu.bh

Key words: multiple sclerosis, demyelination, cuprizone, environment enhancement, depression, water maze, motor coordination, anxiety

\section{Introduction}

Multiple sclerosis (MS) is a disease of the central nervous system (CNS) characterized by multifocal demyelination along with astrocyte gliosis. These processes affect several structures in the CNS, resulting in severe neurological deficits (1). It has been reported that $43-70 \%$ of patients with MS experience cognitive deficits (2). Cognitive impairment in patients with MS is variable depending on the disease stage and type of clinical course (3). MS-associated cognitive impairment is has a negative effect on the quality of life of patients (4), and makes them less likely to be employed or actively engaged in daily activities (5). Although the causes of cognitive impairment in patients MS are currently unclear, depression is believed to be a contributing factor (6-8). Depression often burdens people with MS-related cognitive dysfunction (8), and lifetime prevalence of major depression in MS patients is as high as $50 \%$ (9). Amelioration of depression has been suggested as a mean to improve cognitive functioning in MS. Factors that can contribute to the reduction of depression include social, physical and cognitive stimulation. The effect of these factors is mimicked in laboratory animals housed in an enhanced environment (EE), which has been shown to improve brain development and memory in both normal and pathological conditions $(10,11)$.

The expression of brain-derived neurotrophic factor and tyrosine kinase receptor $\mathrm{B}$, key neuronal differentiation and survival factors, is increased with exposure to EE (12). A host of studies have strengthened the notion that EE is beneficial to a variety of neurodegenerative diseases by protecting against cognitive impairment and improving cognitive performance in Alzheimer's and Huntington's mouse models, respectively (13). Upon studying the effect of EE on experimental autoimmune encephalomyelitis in rodents, a model of MS, results favored functional recovery (14). However, there is insufficient data to draw a conclusion regarding the effect of EE on MS mouse models.

The cuprizone-induced demyelination model has attracted increasing interest during the last decade as a method of inducing MS (15). Indeed, cuprizone-induced demyelination in distinct brain regions, including the corpus callosum, is the most frequently investigated white matter tract in animal 
models $(15,16)$. The addition of $0.2 \%$ cuprizone (bis-cyclohexanone oxaldihydrazone), a copper chelating agent, to the diet of male C57BL/6 strain mice induces spatially and temporally well-defined histopathological alterations in the CNS (17). Furthermore, cuprizone-fed animals in the cuprizone model experience oligodendrocyte death and steady demyelination (18). This demyelination effect was evident 5-6 weeks after cuprizone induction $(18,19)$, whereas remyelination occurred two weeks after the discontinuation of cuprizone. In addition, cuprizone-induced toxicity has been widely used to identify treatments for demyelinating diseases (15,20-22).

The present study investigated the impact of induced depression on a cuprizone mouse model of demyelination and the effectiveness of EE as a method of intervention.

\section{Materials and methods}

Animals. Male C57BL/6 strain mice were bred in the animal lab of Arabian Gulf University. All animal protocols have been approved by the Ethical Committee for Animal Experiments of Arabian Gulf University.

Housing conditions. Three-week-old mice were housed in either standard housing or EE conditions for a period of 9 weeks. Standard cages $(33 \times 15 \times 13 \mathrm{~cm})$, containing 2-3 mice per cage, were cleaned twice a week. All mice were maintained on a 12-h light/dark cycle and fed ad libitum. All tests were performed during the dark cycle.

\section{Experimental design}

Experimental group. Mice were subdivided into five groups, based on the intervention received. A total of 46 mice, dived into 5 groups, were tested: i) Cuprizone mouse model with no intervention (Cup-O; n=9); ii) cuprizone mouse model undergoing induced depression (Cup-Dep; $n=9$ ); iii) cuprizone mouse model housed in an EE (Cup-EE; $n=10)$; iv) cuprizone mouse model housed in an EE and undergoing induced depression (Cup-ED; $n=9)$, and v) a control group, $(n=9)$, which was neither injected with cuprizone nor subjected to depression induction.

Cuprizone model of demyelination. Demyelination was induced by continuously feeding six-week-old C57BL/6 male mice a $0.2 \%$ cuprizone-enriched diet purchased from Specialty Feeds (Western Australia, Australia). After a six-week period of cuprizone diet, all groups were tested. As the drug is temperature sensitive, the food containing the cuprizone was kept at $4^{\circ} \mathrm{C}$ and was replenished every other day. The presence of demyelination in the hippocampus of cuprizone-treated mice was evaluated using Luxol Fast Blue (LFB) staining of formalin-fixed sections as previously described $(23,24)$.

$E E$. Three-week-old mice were housed in an EE, in groups of 5-7 per cage, achieving social stimulation. The EE consisted of two-story cages $(40 \times 26 \times 38 \mathrm{~cm})$, colorful walls, and equipped with a climbing slide and an exercise wheel. The EE method in this study design was based on a previous report by our group (25).

Induced depression. There has not been enough evidence to prove the development of depression (major behavioral defects) in the cuprizone mouse model; therefore, a repeated open-space forced swim test was used to induce chronic depression-like state in mice to mimic the comorbid depression seen in MS patients. The forced swim test is the most commonly used test for the study of depressive-like behavior in rodents (26). It is also considered as a preclinical test to the screening of antidepressant activity in rats and mice (27-29). Mice were made to swim in lukewarm water $\left(32 \pm 2^{\circ} \mathrm{C}\right)$ in an inescapable plastic container $(43 \times 24 \times 23 \mathrm{~cm})$ for $15 \mathrm{~min}$ daily for 4 consecutive days. The container was filled to a depth of $13 \mathrm{~cm}$ water; mice were not able to touch the bottom with their feet or tails. This procedure resembles an inescapable stressor producing an alternation in neural activity and brain cell proliferation that is characteristic of depression. The protocol was initiated 6 days prior to the testing period, as the effect of depression lasts for 3 weeks (30).

Tests. All tests were conducted, scored and analyzed blindly. Morris water maze (MWM) and open field test (OFT) trials were videotaped. Each group was scored by all experimenters to avoid bias.

$M W M$. Spatial learning and memory were tested over a period of 6 days by the MWM (31). The main component of the MWM set up is a circular pool $(140 \times 60 \mathrm{~cm}$, diameter $\mathrm{x}$ height) filled to a depth of $30 \mathrm{~cm}$ water $\left(28 \pm 1^{\circ} \mathrm{C}\right)$. A submerged platform (transparent, round and $8 \mathrm{~cm}$ in diameter) was placed in a fixed location, $1 \mathrm{~cm}$ below water level. Various visible cues were added on the pool's internal walls aiding spatial discrimination. Each mouse was placed in the pool facing one of 5 starting positions (north, south, east, west and southeast), and allowed to swim until it located and climbed onto the submerged platform. Any mouse that failed to locate the platform within $120 \mathrm{sec}$ was placed on the platform by the experimenter. All mice were allowed to stay on the platform for $30 \mathrm{sec}$. The mouse was tracked via a video computer system (Noldus Information Technology, Wageningen, Netherlands). Latency and distance travelled to reach the platform, in addition to swimming velocity, were analyzed using the ANY-maze software on 5 consecutive days. Probe trials were conducted on day 6 , where the platform was removed and time spent in the target quadrant was measured.

Rotarod performance test. Rotarod performance test is used to assess motor function, coordination and balance and consists of a rotating drum with a grooved surface for gripping (32). Mice underwent three, 2-min pre-testing trials at a speed of $4 \mathrm{~m} / \mathrm{min}$. Each mouse had three trials, where one constant speed $(4.5 \mathrm{~m} / \mathrm{min})$ was employed. Latency to fall was recorded.

OFT. The exploration of a novel environment and general locomotor activity were evaluated by the OFT. The open field is a square arena $(44 \times 44 \mathrm{~cm})$ surrounded by $32 \mathrm{~cm}$ high walls. The floor of the arena was divided into 16 even squares. The test apparatus was placed in a dark room and the central four squares were illuminated. Each mouse was tested for three, 10-min trials. Following each trial, the arena was cleaned with $70 \%$ ethanol. Distance travelled and time spent in the central zone were recorded. 
Statistical analysis. All data were presented as the mean \pm standard error of mean. T-test was used to establish the validity of the results when comparing groups for the rotarod performance test. In tests with multiple trials, like the MWM and OFT, a two-way repeated Analysis of Variance (ANOVA) with Tukey's post hoc test, was used to calculate the P-value. Statistical analysis was performed using Microsoft Excel (version 2007). $\mathrm{P}<0.05$ was considered to indicate a statistically significant difference.

\section{Results}

Cuprizone-induced demyelination. Demyelination was induced by continuously feeding six-week-old C57BL/6 male mice a $0.2 \%$ cuprizone-enriched diet. The presence of demyelination was observed in the hippocampus of treated mice based on LFB staining (Fig. 1A and B).

Body weight. The weight of the animals was measured in the beginning of the experiment and 1 week later, to determine the cuprizone effect. The results of the effect of cuprizone administration on the body weight of animals are displayed in Table I. Cuprizone-fed animals exhibited a significant reduction in body weight, when comparing their initial body weight (Cup, 22.2 \pm 0.32 ; Cup-Dep, 22.1 \pm 0.2 ; Cup-EE, $22 \pm 0.24$; Cup-ED, 22.3 \pm 0.17 ) to the weight following a week of cuprizone treatment (Cup 20.9 \pm 0.26 , ANOVA, $\mathrm{F}=11.320$ and $\mathrm{P}=0.0039$; Cup-Dep 21.1 \pm 0.2 , ANOVA, $\mathrm{F}=12.462$ and $\mathrm{P}=0.0023$; Cup-EE 21.1 \pm 0.31 , ANOVA, $\mathrm{F}=5.224$ and $\mathrm{P}=0.0362$; Cup-ED 21.3 \pm 0.17 , ANOVA, $\mathrm{F}=18$ and $\mathrm{P}=0.00062$ ). The control animals exhibited a significant increase in weight (from $22.3 \pm 0.23$ vs. $23.03 \pm 0.21$; ANOVA, $\mathrm{F}=5.8255$ and $\mathrm{P}=0.02815$ )

\section{MWM}

Latency and distance travelled to reach the platform. Upon measuring the latency to reach the platform on day 5 , significant results were obtained. All cuprizone groups had worse results than the control group. Cup-EE did significantly better than Cup-O (42.0 \pm 8.2 vs. $67.7 \pm 7.8 \mathrm{sec}$, respectively; ANOVA, $\mathrm{F}=43.788$ and $\mathrm{P}<0.0001)$. Cup-Dep did significantly worse than Cup-O (113.9 \pm 3.5 vs. $67.7 \pm 7.8 \mathrm{sec}$; ANOVA, $\mathrm{F}=29.517$ and $\mathrm{P}<0.001)$ and Cup-ED $(66.5 \pm 7 \mathrm{sec}$; ANOVA, $\mathrm{F}=31.916$ and $\mathrm{P}<0.0001)$, with the latter two groups having comparable results (ANOVA, F=0.0121 and $\mathrm{P}=0.9128$; Fig. 2A). Similar results were obtained with regards to distance travelled to reach the platform on day 5 (Fig. 2B).

Swimming velocity. All cuprizone groups exhibited a slower swimming velocity than the control group on days $1-4$; which was also reflected on the latency results. The swimming velocity of cuprizone groups varied during the testing period with no identifiable pattern (Fig. 2C). Similar swimming velocities indicate that the results were not influenced by differences in muscle power among the mice of the cuprizone groups. No significant differences in swimming velocity were observed between the groups $(\mathrm{P}>0.05)$.

Percentage of time spent in the target quadrant. Apart from the control group, which spent $42.9 \pm 3 \%$ of the time

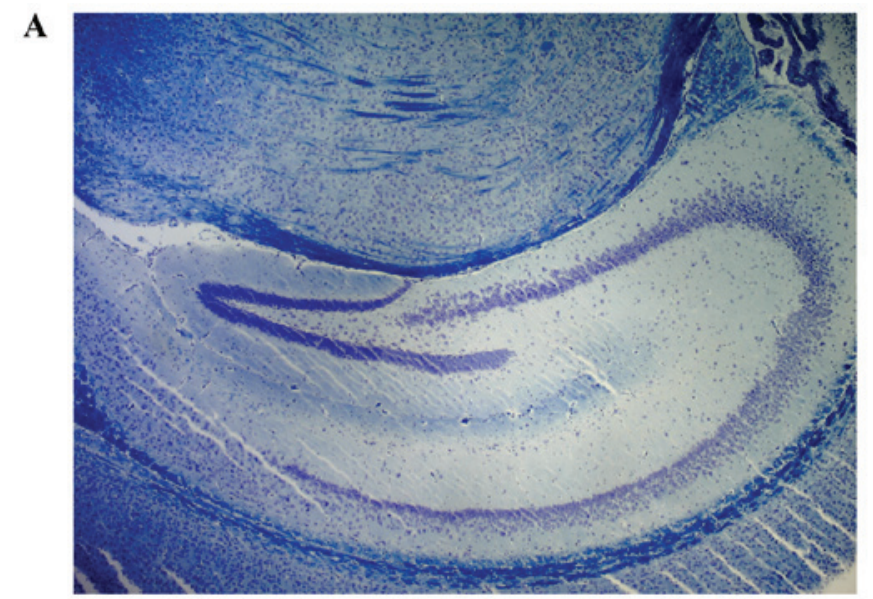

B

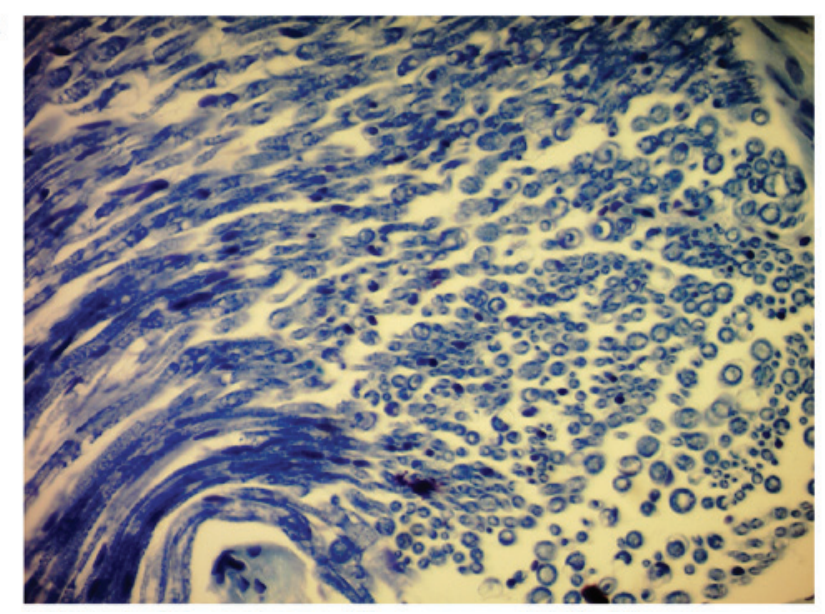

Figure 1. Hippocampal demyelination in cuprizone-treated mice. Mice were treated with cuprizone-enriched diet. Cross-sections (5 $\mu \mathrm{m}$ thick) from hippocampus were stained for myelin with LFB. (A) Hippocampal demyelination (magnification, x400). (B) Hippocampal demyelination (magnification, x100).

in the target quadrant, none of the other groups exhibited a learning behavior, since they spent $<25 \%$ of the time in the target quadrant. However, a variation was noted among cuprizone groups. Cup-EE (19.2 $\pm 2.3 \%)$ had significantly better results than Cup-Dep (11.1 $\pm 2.5 \%$; ANOVA, F=5.8964 and $\mathrm{P}=0.01721)$ and Cup-O (12.3 $\pm 3.1 ;$ ANOVA, $\mathrm{F}=5.1110$ and $\mathrm{P}=0.026$ ), whereas no significant difference was observed between Cup-O and Cup-ED (13.3 \pm 2.9 ; ANOVA, $\mathrm{F}=0.0596$, and $\mathrm{P}=0.8076$; Fig. 2D).

\section{Rotarod performance test}

Latency to fall. At the speed of $4.5 \mathrm{~m} / \mathrm{min}$, no significant difference was observed between the control $(70.1 \pm 10.6 \mathrm{sec})$ and Cup-EE $(80.1 \pm 15.7 \mathrm{sec} ; \mathrm{ANOVA}, \mathrm{F}=0.2799$ and $\mathrm{P}=0.599)$. A variation was noted among cuprizone groups; Cup-EE mice spent significantly more time on the rotating rod than Cup-O mice $(80.1 \pm 15.7$ vs. $32.2 \pm 8.7$, respectively; ANOVA, $\mathrm{F}=7.1026$ and $\mathrm{P}=0.0102)$. The results of the Cup-Dep group were significantly worse than those of Cup-O (13.3 \pm 1.8 vs. $32.2 \pm 8.7$; ANOVA, $\mathrm{F}=4.594$ and $\mathrm{P}=0.0368$ ), indicating declined motor functioning. No significant difference was observed between the Cup-ED and Cup-O groups $(31.0 \pm 6.1$ vs. $32.2 \pm 8.7$; ANOVA, $\mathrm{F}=0.0133$ and $\mathrm{P}=0.909$; Fig. 3). 
Table I. The effect of cuprizone administration on the body weight of animals.

\begin{tabular}{lccc}
\hline Group & $\begin{array}{c}\text { Weight }(\mathrm{gm}) \text { at the } \\
\text { start of experiment }\end{array}$ & $\begin{array}{c}\text { Weight (gm) after one } \\
\text { week of cuprizone }\end{array}$ & \multicolumn{2}{c}{ P-value } \\
\hline Control (no cuprizone injection) & $22.3 \pm 0.23$ & $23.03 \pm 0.21$ & 0.00390 .26 \\
Cup & $22.2 \pm 0.32$ & $21.1 \pm 0.2$ & 0.0027 \\
Cup-Dep & $22.1 \pm 0.2$ & $21.1 \pm 0.31$ & 0.0362 \\
Cup-EE & $22 \pm 0.24$ & $21.3 \pm 0.17$ & 0.0006 \\
Cup-ED & $22.3 \pm 0.17$ & &
\end{tabular}

Data are presented as mean \pm standard error of mean (SEM). Cup, cuprizone; Cup-Dep, cuprizone underwent depression; Cup-EE, cuprizone housed in EE; Cup-ED, cuprizone housed in EE and underwent depression.

A
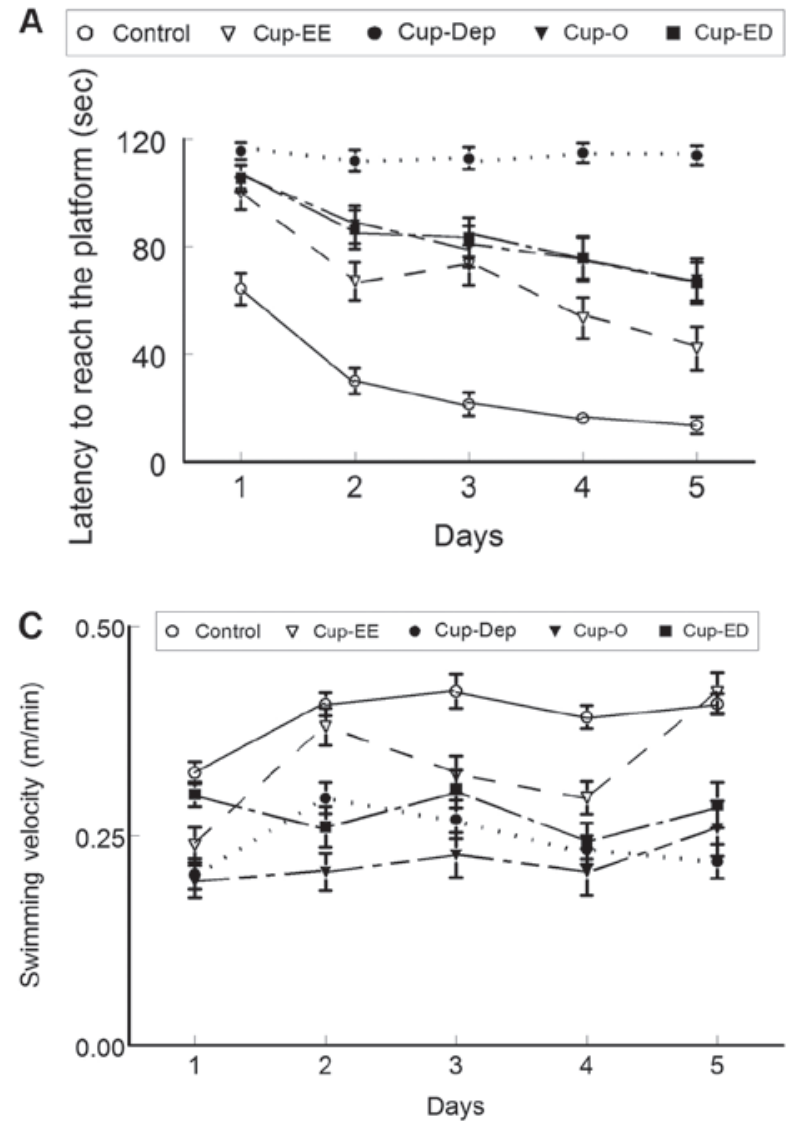

B $\bigcirc$ Control $\nabla$ Cup-EE $\bullet$ Cup-Dep $\nabla$ Cup-O a Cup-ED
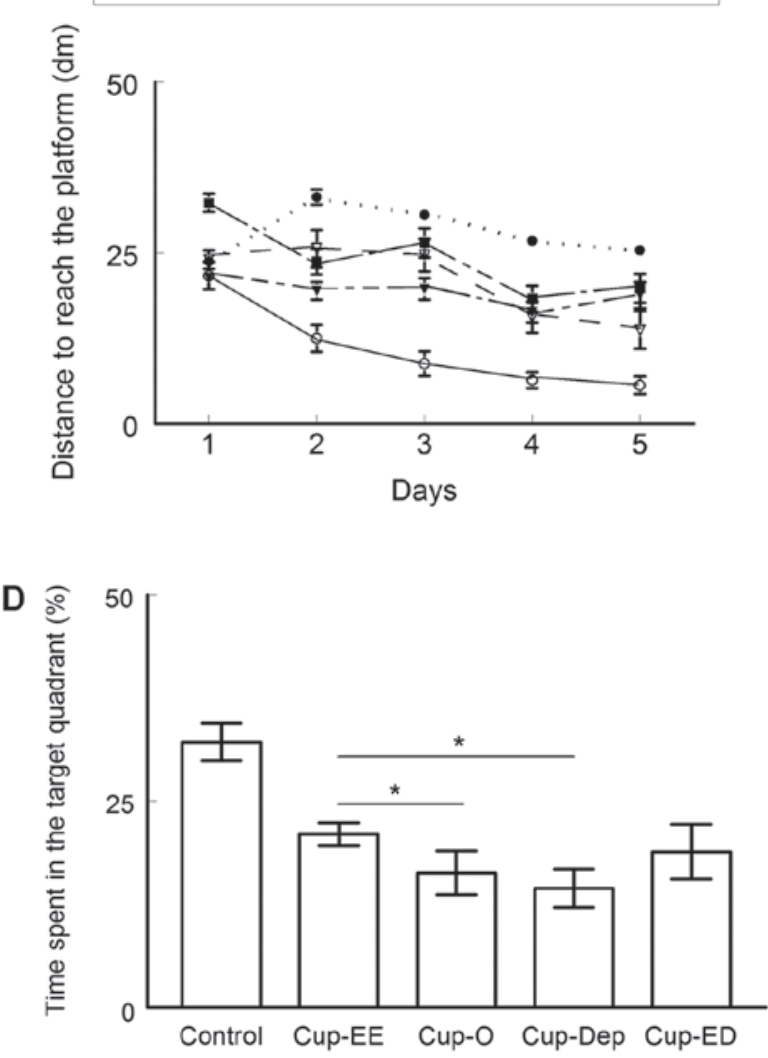

Figure 2. MWM. The cuprizone mouse model showed different levels of cognitive deficits based on the method of intervention. (A) Latency (sec) to reach the platform. (B) distance (dm) travelled to reach the platform throughout testing period (days). On day 5, cuprizone groups performed significantly worse than the control, as they had increased latencies and travelled a longer distance to reach the platform. Cup-EE performed better than the other cuprizone groups on day $5(\mathrm{P}<0.0001)$. Cup-Dep had the highest latency throughout the testing period and travelled the longest distance on days $2-5$ ( $<0.001)$. Cup-ED exhibited similar latencies and distance travelled to Cup-O (P>0.05). (C) Swimming velocity ( $\mathrm{m} / \mathrm{sec}$ ) during testing period (days). The control group was relatively faster than the cuprizone group on days 1-4. Swimming velocity in the cuprizone groups varied during the testing period. No significant differences were observed among the groups $(\mathrm{P}>0.05)$. (D) Bar graph depicting the percentage of time spent in the target quadrant during the probe trial. None of the cuprizone groups exhibited a learning behavior, as they spent $<25 \%$ of the time in the target quadrant. Cup-EE spent significantly more time in the target quadrant than Cup-Dep and Cup-O ( $\mathrm{P}<0.05)$, indicating better performance. The results of Cup-ED were comparable to those of Cup-O (P>0.05). MWM, Morris water maze.

\section{Open field}

Time spent in the central zone. OFT results showed no anxiety-like behavior in any of the groups, as they spent a comparable amount of time in the central zone. No significant difference was observed between the control and cuprizone groups ( $\mathrm{P}>0.05$; Fig. 4).

\section{Discussion}

MS is a neurological chronic inflammatory disorder of the CNS characterized by multifocal demyelination along with astrocyte gliosis (1). Impaired cognition and motor activity are commonly encountered in MS patients (3) and depression is 


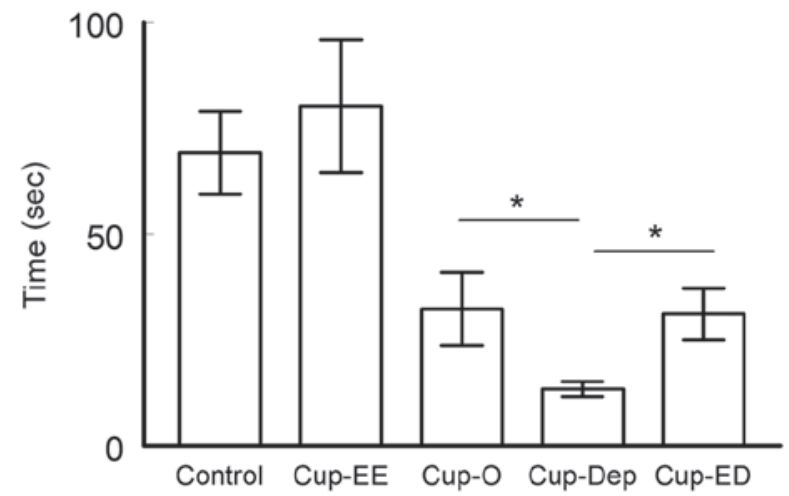

Figure 3. Rotarod performance test as a measure of motor coordination. Bar graph plots the time (sec) spent on the rotating rod when tested at a constant speed $(4.5 \mathrm{~m} / \mathrm{min})$. No significant difference was observed between the control and Cup-EE groups ( $\mathrm{P}>0.05)$. Cup-EE spent significantly more time on the rotating rod than other cuprizone groups $\left({ }^{*} \mathrm{P}<0.05\right)$, indicating that EE improved motor coordination. Cup-Dep had significantly worse results than both Cup-O and cup-ED ( $\mathrm{P}<0.05)$. EE, enhanced environment.

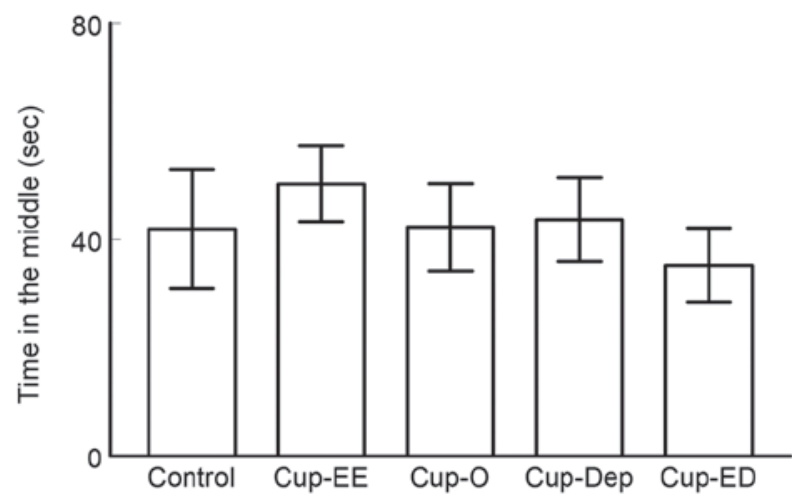

Figure 4. Behavioral changes measured by OFT. Bar graph illustrating the time spent in the central zone (sec) of the arena. All groups spent comparable time in the central zone without any significant differences observed among the control and cuprizone groups $(\mathrm{P}>0.05)$. This indicates the absence of anxiety-like behavioral changes in the cuprizone groups. OFT, open field test.

believed to be a contributing factor (6-9). Cuprizone-induced demyelination is considered a well-established model of MS in animals $(15,16)$, which leads to oligodendrocyte apoptosis and steady demyelination and histopathological alterations in the CNS $(17,18)$. Cuprizone-induced toxicity has been widely used to identify treatments for demyelinating diseases (20-22).

The current study investigated the impact of induced depression on a cuprizone mouse model of demyelination and the effectiveness of EE as a method of intervention. C57BL/6 male mice were divided into five groups: Cup-O, Cup-Dep, Cup-EE,Cup-ED and the control group. In the present study, we first confirmed the presence of demyelination in our cuprizonetreated mice using LFB staining, a commonly used method to observe myelin under the light to detect demyelination in the CNS $(23,24)$. Following a week of cuprizone treatment, our results showed a significant reduction in the body weight of the animals, indicating the effectiveness of cuprizone on these animals (33). The impact of induced depression was studied in terms of cognitive and motor function. We tested the spatial learning and memory in MWM as well as motor learning and coordination by rotarod test. In the cuprizone model, induced depression further worsened cognition, when compared to the non-intervention group, as demonstrated by the results of the MWM. Of note, further experiments can be conducted on transgenic and knockout mice to ensure that abnormal responses, particularly in learning and memory tasks, are indeed due to cognitive motor impairment. Moreover, experiments using nutritional interventions such as anti-oxidants-rich food in the diet of animals could be conducted to slow the progression of cognitive decline.

Depression has been studied as an independent factor influencing cognition and general outcome in MS patients. A study conducted on MS patients with various degrees of depression revealed greater neuropsychological dysfunction, as compared to non-depressed MS patients (34). Moreover, psychotherapy has been suggested as a method of decreasing cognitive impairments in MS patients (35).

As established by the results of the rotarod performance test in the present study, induced-depression appeared to cause a significant decline in the motor performance of animals, when compared to those undergoing no intervention.

Animals exposed to an EE exhibited enhanced stimulation in their sensory, motor and cognitive systems, when compared to those under normal housing conditions (36). The components of EE have been proven to enhance neurogenesis, which in turn reflects on motor function and cognition (12).

While in the present study EE did not rescue the cognitive deficits in the cuprizone mouse model, enriched mice performed significantly better, as compared to other cuprizone groups. Altogether, the results of the MWM in this study provided evidence that EE improved spatial learning and memory in the MS animal model. Similarly, EE significantly improved motor function in the cuprizone mouse model, as evidenced by the ability of the mice to spend more time on the rotating rod. Notably, the effect of EE was more pronounced in motor activity, suggesting that spatial learning and memory are more complex processes.

The present findings revealed a marked effect of EE on mice in the cuprizone group undergoing induced depression. A complete reversal of the effects of induced depression was noted in terms of cognition and motor function. Mice in the cuprizone group undergoing induced depression and exposed to EE performed similarly to those from the cuprizone group with no intervention. The aforementioned results suggested that, although EE exposure did not regain control values in the cuprizone mouse model of demyelination, it certainly reversed the effect of induced depression. These findings indicated that the disease effects of MS on its own could not be reversed by $\mathrm{EE}$, but the additive effect of induced depression could. Nevertheless, the underlying biological mechanism of EE in the cuprizone-induced demyelination mouse model needs to be further investigated. For example, genetic studies including the expression of genes and receptors that influence brain plasticity and function may be important to elucidate the effect of changes in gene expression on EE in cuprizone-induced demyelination in animal models.

In the present study, the performance of the Cup-Dep group of animals in the MWM with a hidden platform was significantly worse than that of all other groups. This deficit was unlikely, due to a learned helplessness that may have been induced by the 
forced swim test, since the Cup-ED group completely reversed the effects of depression in the MWM. In addition, the performance of the Cup-ED group in a maze with a visible platform (data not shown) was indistinguishable from that of other groups. Although all cuprizone groups in the present study exhibited no learning behavior, induced depression further deteriorated performance, which was ameliorated by EE.

Considerable evidence has suggested that MS patients have higher rates of anxiety $(37,38)$. In the present study, the results of the OFT failed to show an anxiety-like behavior in the cuprizone mouse model, since all groups exhibited similar exploratory behavior. Similar results, suggesting that anxietylike behavioral changes are subtle or absent in the animal model of MS, were reported by Rodrigues et al (39).

EE and depression are known to induce molecular and cellular changes in specific brain regions $(40,41)$. These changes included altered gene expression profiles and enhanced neurogenesis and synaptic plasticity (40).

Long term potentiation (LTP) and long-term depression (LTD), two opposing forms of long-term plasticity, are believed to be the actual synaptic processes underlying learning and memory (42). EE modulates synaptic plasticity by enhancing LTP, thus improving memory (25). Conversely, depression and stressful conditions impair LTP and facilitate LTD (41).

These observations correlate possible synaptic plasticity changes in the hippocampus with exposure to different factors. The present study suggests synaptic plasticity involvement in the cuprizone mouse model of demyelination, where memory was modulated upon exposure to EE and depression.

Increasing evidence suggests that the decline in daily activities and functions in MS patients is the result of interplay, among several other factors including depression which, however, is not solely caused by the disease. These findings clearly demonstrated the deteriorating effects of depression on the cuprizone mouse model, and the ameliorating effect of $\mathrm{EE}$ on the decline in cognition and motor function. Data from the present study are very encouraging in the context of using EE to improve the quality of life of patients with MS.

A limitation of the present study was the lack of morphological and biochemical markers to prove demyelination. However, cuprizone-induced demyelination is a widely used model of MS in animals $(15,16)$.

In conclusion, the results of this study raise the possibility of future applications of non-medical therapy for MS, and encourage future researchers to explore the technological advances of non-invasive manipulations of brain activity to modulate the course of various neurological diseases.

\section{Acknowledgements}

The authors would like to thank the technical staff of the animal house at the College of Medicine and Medical Sciences, Arabian Gulf University.

\section{Funding}

The present study was supported by departmental fund from the Department of Physiology, College of Medicine and Medical Sciences, Arabian Gulf University (grant no. 20-16).

\section{Availability of data and materials}

The datasets used and/or analyzed during the present study are available from the corresponding author on reasonable request.

\section{Authors' contributions}

AM, GA, AK developed the project and edited the manuscript; AM, AAlma, ZA, HA, RA, FA, AAlmu, AAl-M, AK collected the data; all authors performed data analysis, managed the data and wrote the manuscript. The final version of the manuscript has been read and approved by all authors.

\section{Ethical approval and consent to participate}

The study received ethical approval from the Research and Ethics Committee of the College of Medicine and Medical Sciences (Arabian Gulf University, Manama, Kingdom of Bahrain).

\section{Patient consent for publication}

Not applicable.

\section{Competing interests}

The authors declare that they have no competing interests.

\section{References}

1. Beer S, Khan F and Kesselring J: Rehabilitation interventions in multiple sclerosis: An overview. J Neurol 259: 1994-2008, 2012.

2. Langdon DW, Amato MP, Boringa J, Brochet B, Foley F, Fredrikson S, Hämäläinen $\mathrm{P}$, Hartung HP, Krupp L, Penner IK, et al: Recommendations for a Brief International Cognitive Assessment for Multiple Sclerosis (BICAMS). Mult Scler 18: 891-898, 2012

3. Amato MP, Zipoli V and Portaccio E: Multiple sclerosis-related cognitive changes: A review of cross-sectional and longitudinal studies. J Neurol Sci 245: 41-46, 2006

4. Cioncoloni D, Innocenti I, Bartalini S, Santamecchi E, Rossi S Rossi A and Ulivelli M: Individual factors enhance poor healthrelated quality of life outcome in multiple sclerosis patients. Significance of predictive determinants. J Neurol Sci 345: 213-219, 2014.

5. Salter A, Thomas N, Tyry T, Cutter G and Marrie RA: Employment and absenteeism in working-age persons with multiple sclerosis. J Med Econ 20: 493-502, 2017.

6. Raimo S, Trojano L, Pappacena S, Alaia R, Spitaleri D, Grossi D and Santangelo G: Neuropsychological correlates of theory of mind deficits in patients with multiple sclerosis. Neuropsychology 31: 811-821, 2017.

7. Gay MC, Bungener C, Thomas S, Vrignaud P, Thomas PW Baker R, Montel S, Heinzlef O, Papeix C, Assouad R and Montreuil M: Anxiety, emotional processing and depression in people with multiple sclerosis. BMC Neurol 17: 43, 2017.

8. Rahn K, Slusher B and Kaplin A: Cognitive impairment in multiple sclerosis: A forgotten disability remembered. Cerebrum 2012: 14, 2012

9. Siegert RJ and Abernethy DA: Depression in multiple sclerosis: A review. J Neurol Neurosurg Psychiatry 76: 469-475, 2005.

10. Kazlauckas V, Pagnussat N, Mioranzza S, Kalinine E, Nunes F, Pettenuzzo L, Souza DO, Portela LV, Porciúncula LO and Lara DR: Enriched environment effects on behavior, memory and BDNF in low and high exploratory mice. Physiol Behav 102: 475-480, 2011.

11. Zhao YY, Shi XY, Qiu X, Lu W, Yang S, Li C, Chen L, Zhang L, Cheng GH and Tang Y: Enriched environment increases the myelinated nerve fibers of aged rat corpus callosum. Anat Rec (Hoboken) 295: 999-1005, 2012. 
12. Cao W, Duan J, Wang X, Zhong X, Hu Z, Huang F, Wang H, Zhang J, Li F, Zhang J, et al: Early enriched environment induces an increased conversion of proBDNF to BDNF in the adult rat's hippocampus. Behav Brain Res 265: 76-83, 2014.

13. Wood NI, Carta V, Milde S, Skillings EA, McAllister CJ, Ang YL, Duguid A, Wijesuriya N, Afzal SM, Fernandes JX, et al: Responses to environmental enrichment differ with sex and genotype in a transgenic mouse model of huntington's disease. PLoS One 5: e9077, 2010.

14. Magalon K, Cantarella C, Monti G, Cayre M and Durbec P: Enriched environment promotes adult neural progenitor cell mobilization in mouse demyelination models. Eur J Neurosci 25 761-771, 2007.

15. Kipp M, Clarner T, Dang J, Copray S and Beyer C: The cuprizone animal model: New insights into an old story. Acta Neuropathol 118: 723-736, 2009.

16. Torkildsen O, Brunborg LA, Myhr KM and Bø L: The cuprizone model for demyelination. Acta Neurol Scand Suppl 188: 72-76, 2008.

17. Kipp M, Nyamoya S, Hochstrasser T and Amor S: Multiple sclerosis animal models: A clinical and histopathological perspective. Brain Pathol 27: 123-137, 2017.

18. Komoly S: Experimental demyelination caused by primary oligodendrocyte dystrophy. Regional distribution of the lesions in the nervous system of mice [corrected]. Ideggyogy Sz 58: 40-43, 2005

19. Stidworthy MF, Genoud S, Suter U, Mantei N and Franklin RJ: Quantifying the early stages of remyelination following cuprizone-induced demyelination. Brain Pathol 13: 329-339, 2003.

20. Matsushima GK and Morell P: The neurotoxicant, cuprizone, as a model to study demyelination and remyelination in the central nervous system. Brain Pathol 11: 107-116, 2001.

21. Morell P, Barrett CV, Mason JL, Toews AD, Hostettler JD, Knapp GW and Matsushima GK: Gene expression in brain during cuprizone-induced demyelination and remyelination. Mol Cell Neurosci 12: 220-227, 1998.

22. Skripuletz T, Gudi V, Hackstette D and Stangel M: De-and remyelination in the CNS white and grey matter induced by cuprizone: The old, the new, and the unexpected. Histol Histopathol 26 : 1585-1597, 2011.

23. Dumitrascu OM, Mott KR and Ghiasi H: A comparative study of experimental mouse models of central nervous system demyelination. Gene Ther 21: 599-608, 2014

24. Ingram G, Loveless S, Howell OW, Hakobyan S, Dancey B, Harris CL, Robertson NP, Neal JW and Morgan BP: Complement activation in multiple sclerosis plaques: An immunohistochemical analysis. Acta Neuropathol Commun 2 53, 2014.

25. Artola A, von Frijtag JC, Fermont PC, Gispen WH, Schrama LH, Kamal A and Spruijt BM: Long-lasting modulation of the induction of LTD and LTP in rat hippocampal CA1 by behavioura stress and environmental enrichment. Eur J Neurosci 23 261-272, 2006

26. Yankelevitch-Yahav R, Franko M, Huly A and Doron R: The forced swim test as a model of depressive-like behavior. J Vis Exp: 2 Mar, 2015 doi: 10.3791/52587.
27. Castagné V, Moser P and Porsolt RD: Behavioral assessment of antidepressant activity in rodents. Methods of behavior analysis in neuroscience. 2nd edition. Boca Raton (FL): CRCPress/Taylor \& Francis, 2009

28. Porsolt RD, Le Pichon M and Jalfre M: Depression: A new animal model sensitive to antidepressant treatments. Nature 266: 730-732, 1977.

29. Porsolt RD, Bertin A and Jalfre M: 'Behavioural despair' in rats and mice: Strain differences and the effects of imipramine. Eur J Pharmacol 51: 291-294, 1978.

30. Stone EA, Lin Y and Quartermain D: Evaluation of the repeated open-space swim model of depression in the mouse. Pharmacol Biochem Behav 91: 190-195, 2008.

31. D'Hooge R and De Deyn PP: Applications of the Morris water maze in the study of learning and memory. Brain Res Brain Res Rev 36: 60-90. 2001.

32. Bohlen M, Cameron A, Metten P, Crabbe JC and Wahlsten D: Calibration of rotational acceleration for the rotarod test of rodent motor coordination. J Neurosci Methods 178: 10-14, 2009.

33. Sachs HH, Bercury KK, Popescu DC, Narayanan SP and Macklin WB: A new model of cuprizone-mediated demyelination/remyelination. ASN Neuro 6: pii: 1759091414551955, 2014.

34. Boeschoten RE, Braamse AMJ, Beekman ATF, Cuijpers P, van Oppen P, Dekker J and Uitdehaag BMJ: Prevalence of depression and anxiety in Multiple Sclerosis: A systematic review and metaanalysis. J Neurol Sci 372: 331-341, 2017.

35. Bilgi E, Özdemir HH, Bingol A and Bulut S: Evaluation of the effects of group psychotherapy on cognitive function in patients with multiple sclerosis with cognitive dysfunction and depression. Arq Neuropsiquiatr 73: 90-95, 2015.

36. Alwis DS and Rajan R: Environmental enrichment and the sensory brain: The role of enrichment in remediating brain injury. Front Syst Neurosci 8: 156, 2014.

37. Serra-de-Oliveira N, Boilesen SN, Prado de França Carvalho C, LsSueur-Maluf L, Zollner Rde L, Spadari RC, Medalha CC and Monteiro de Castro G: Behavioural changes observed in demyelination model shares similarities with white matter abnormalities in humans. Behav Brain Res 287: 265-275, 2015.

38. Acharjee S, Nayani N, Tsutsui M, Hill MN, Ousman SS and Pitman QJ: Altered cognitive-emotional behavior in early experimental autoimmune encephalitis-Cytokine and hormonal correlates. Brain Behav Immun 33: 164-172, 2013.

39. Rodrigues DH, Vilela Mde C, Lacerda-Queiroz N, Miranda AS, Sousa LF, Reis HJ and Teixeira AL: Behavioral investigation of mice with experimental autoimmune encephalomyelitis. Arq Neuropsiquiatr 69: 938-942, 2011.

40. Spires TL and Hannan AJ: Nature, nurture and neurology: Geneenvironment interactions in neurodegenerative disease. FEBS Anniversary Prize Lecture delivered on 27 June 2004 at the 29th FEBS Congress in Warsaw. FEBS J 272: 2347-2361, 2005.

41. Von Frijtag JC, Kamal A, Reijmers LG, Schrama LH, van den Bos R and Spruijt BM: Chronic imipramine treatment partially reverses the long-term changes of hippocampal synaptic plasticity in socially stressed rats. Neurosci Lett 309: 153-156, 2001.

42. Bliss TV and Cooke SF: Long-term potentiation and long-term depression: A clinical perspective. Clinics (Sao Paulo) 66 (Suppl 1): S3-S17, 2011. 